\title{
Isomer-delayed gamma-ray spectroscopy of neutron-rich ${ }^{166} \mathrm{~Tb}$
}

\author{
L.A. Gurgi ${ }^{1 a}$, P.H. Regan ${ }^{1,2}$, P.-A. Söderström ${ }^{3}$, H. Watanabe ${ }^{4,5}$, P.M. Walker ${ }^{1}$, Zs. Podolyák ${ }^{1}$, S. Nishimura ${ }^{3}$,
} T.A. Berry ${ }^{1}$, P. Doornenbal ${ }^{3}$, G. Lorusso ${ }^{1,2,3}$, T. Isobe ${ }^{3}$, H. Baba ${ }^{3}$, Z.Y. Xu ${ }^{6,7}$, H. Sakurai ${ }^{3,8}$, T. Sumikama ${ }^{3,9}$, W.N. Catford ${ }^{1}$, A.M. Bruce ${ }^{10}$, F. Browne ${ }^{10}$, G.J. Lane ${ }^{11}$, F.G. Kondev ${ }^{12}$, A. Odahara ${ }^{13}$, J. Wu ${ }^{3,14}$, H.L. Liu ${ }^{15}$, F.R. Xu ${ }^{14}$, Z. Korkulu ${ }^{3,16}$, P. Lee ${ }^{17}$, J.J. Liu ${ }^{16}$, V.H. Phong ${ }^{3,18}$, A. Yagi ${ }^{13}$, G.X. Zhang ${ }^{15}$, T. Alharbi ${ }^{19}$, R.J. Carroll ${ }^{1}$, K.Y. Chae ${ }^{20}$, Zs. Dombradi ${ }^{16}$, A. Estrade ${ }^{7,21}$, N. Fukuda ${ }^{3}$, C. Griffin ${ }^{21}$, E. Ideguchi ${ }^{13,23}$, N. Inabe ${ }^{3}$, H. Kanaoka ${ }^{13}$, I. Kojouharov ${ }^{24}$, T. Kubo ${ }^{3}$, S. Kubono $^{3}$, N. Kurz ${ }^{24}$, I. Kuti ${ }^{16}$, S. Lalkovski ${ }^{1}$, E.J. Lee ${ }^{20}$, C.S. Lee ${ }^{17}$, G. Lotay ${ }^{1}$, C.B. Moon ${ }^{25}$, I. Nishizuka ${ }^{9}$, C.R. Nita ${ }^{10,26}$, Z. Patel ${ }^{1}$, O.J. Roberts ${ }^{27}$, H. Schaffner ${ }^{24}$, C.M. Shand ${ }^{1}$, H. Suzuki ${ }^{3}$, H. Takeda ${ }^{3}$, S. Terashima ${ }^{5}$, Zs. Vajta ${ }^{16}$, S. Kanaya ${ }^{13}$, and J.J. Valiente-Dobòn ${ }^{28}$

1 Department of Physics, University of Surrey, Guildford GU2 7XH, UK

2 National Physical Laboratory, Teddington, 11 0LW, UK

RIKEN Nishina Center, 2-1 Hirosawa, Wako-shi, Saitama 351-0198, Japan

4 International Research Center for Nuclei and Particles in the Cosmos, Beihang University, Beijing 100191, China

School of Physics and Nuclear Energy Engineering, Beihang University, Beijing 100191, China

Department of Physics, the University of Hong Kong, Pokfulam Road, Hong Kong

KU Leuven, Instituut voor Kern- en Stralings fysica, 3001 Leuven, Belgium

8 Department of Physics, University of Tokyo, Hongo, Bunkyo-ku, Tokyo 113-0033, Japan

9 Department of Physics, Tohoku University, Aoba, Sendai, Miyagi 980-8578, Japan

10 School of Computing, Engineering and Mathematics, University of Brighton, BN2 4JG, UK

11 Department of Nuclear Physics, R.S.P.E., Australian National University, Canberra, A.C.T.02000, Australia

12 Nuclear Engineering Division, Argonne National Laboratory, Argonne, Illinois 60439, USA

13 Department of Physics, Osaka University, Machikaneyama-machi 1-1, Osaka 560-0043, Toyonaka, Japan

14 School of Physics, Peking University, Beijing 100871, China

15 Department of Applied Physics, School of Science, Xi'an Jiaotong University, China

16 Institute for Nuclear Research, Hungarian Academy of Sciences, PO Box 51, Debrecen, Hungary

17 Department of Physics, Chung-Ang University, Seoul 156-756, Republic of Korea

18 NU Hanoi University of Science, 334 Nguyen Trai, Thanh Xuan, Hanoi, Vietnam

19 Department of Physics, College of Science in Zulfi, Almajmaah University, PO Box 1712, 11932, Saudi Arabia

20 Department of Physics, Sungkyunkwan University, Suwon 440-746, Republic of Korea

21 School of Physics and Astronomy, University of Edinburgh, Edinburgh EH9 3JZ, UK

22 Department of Physics, Central Michigan University, Mount Pleasant, Michigan 48859, USA

23 Research Center for Nuclear Physics (RCNP), Osaka University, Ibaraki, Osaka 567-0047, Japan

${ }^{24}$ GSI Helmholtzzentrum für Schwerionenforschung GmbH, 64291 Darmstadt, Germany

25 Hoseo University, Asan, Chungnam 336-795, Korea

${ }^{26}$ Horia Hulubei National Institute of Physics and Nuclear Engineering,(IFIN-HH), 077125 Bucharest, Romania

27 School of Physics, University College Dublin, Belfield, Dublin 4, Ireland

28 Istituto Nazionale di Fisica Nucleare, Laboratori Nazionali di Legnaro, 35020 Legnaro, Italy

\begin{abstract}
This short paper presents the identification of a metastable, isomeric-state decay in the neutron-rich odd-odd, prolate-deformed nucleus ${ }^{166} \mathrm{~Tb}$. The nucleus of interest was formed using the in-flight fission of a $345 \mathrm{MeV}$ per nucleon ${ }^{238} \mathrm{U}$ primary beam at the RIBF facility, RIKEN, Japan. Gamma-ray transitions decaying from the observed isomeric states in ${ }^{166} \mathrm{~Tb}$ were identified using the EURICA gamma-ray spectrometer, positioned at the final focus of the BigRIPS fragments separator. The current work identifies a single discrete gamma-ray transition of energy $119 \mathrm{keV}$ which de-excites an isomeric state in ${ }^{166} \mathrm{~Tb}$ with a measured half-life of 3.5(4) $\mu \mathrm{s}$. The multipolarity assignment for this transition is an electric dipole and is made on the basis internal conversion and decay lifetime arguments. Possible two quasi-particle Nilsson configurations for the initial and final states which are linked by this transition in ${ }^{166} \mathrm{~Tb}$ are made on the basis of comparison with Blocked BCS Nilsson calculations, with the predicted ground state configuration for this nucleus arising from the coupling of the $\nu \frac{1}{2}^{-}[521]$ and $\pi \frac{3}{2}^{+}$[411] Nilsson orbitals.
\end{abstract}

\footnotetext{
a e-mail: 1.a.gurgi@surrey.ac.uk
}

(C) The Authors, published by EDP Sciences. This is an Open Access article distributed under the terms of the Creative Commons Attribution License 4.0 (http://creativecommons.org/licenses/by/4.0/). 


\section{Introduction}

The valence maximum nucleus ${ }^{170} \mathrm{Dy}_{1} 04$ lies in the centre of the deformed region of prolate nuclear rotors [1]. The particular details of which nuclear orbitals lie close to the Fermi surface and how they influence the evolution of nuclear shapes can be studied by investigating the structure of odd-A and odd-odd nuclei close to the even-even valence maximum core. The present work presents information on the possible single-particle structures which are formed via proton-neutron deformed Nilsson orbital couplings in the $\mathrm{Z}=65, \mathrm{~N}=101$ nucleus ${ }^{166} \mathrm{~Tb}_{1} 01$. Prior to this work, the only information available was on the excited states of ${ }^{166} \mathrm{~Tb}$ were identified following the $\beta^{-}$decay of the ${ }^{166} \mathrm{Gd}$ mother nucleus, in which discrete energy gamma-ray transitions with energies 40 , $119,158,536,976$ and $1016 \mathrm{keV}$ identified [2].

\section{Experimental details, analysis and results}

Neutron-rich nuclei in the vicinity ${ }^{170} \mathrm{Dy}$ were produced following the projectile fission of a $345 \mathrm{MeV}$ per nucleon ${ }^{238} \mathrm{U}$ primary beam on a $2 \mathrm{~mm}$ thick berylium production target at the Radioactive Isotope Beam Factory (RIBF) [3], RIKEN, Japan. The typical primary beam current was $10 \mathrm{pnA}$ and the produced fission fragments were transported and separated on an event-by-event basis using the BigRIPS fragment separator at RIKEN [4,5]. The transported ions were identified event-by-event through the separator using the measured magnetic rigidity $(B \rho)$, Time-of-Flight (ToF) and energy loss $(\Delta \mathrm{E})$ parameters.

The secondary beam fragments were finally brought to rest in the Wide-range Active Silicon Strip Stopper Array (WAS3ABI) [6] which was placed at the final focus of the BigRIPS separator. This position sensitive detector allowed direct correlations with individual implanted ions and also with subsequent $\beta$-decay events in the same location of the stopper arising from the same ion [1]. Discrete-energy gamma-ray decays emitted from the implaned ions (following either isomeric decay or $\beta^{-}$ decay) were measured using the Euroball RIKEN Cluster Array (EURICA) consisting of 84 coaxial high-purity germanium (HPGe) detectors, arranged in an array of $12 \times 7$ element CLUSTER detector modules, complemented by 18 additional $\mathrm{LaBr}_{3}(\mathrm{Ce})$ detectors for fasttiming measurements $[1,7-10]$. Related studies from the same experiment have been reported for isomeric and decay spectroscopy of ${ }^{170}$ Dy and ${ }^{172}$ Dy $[1,10]$.

Two magnetic rigidity $(B \rho)$ settings for the BigRIPS separator were used in the current work. The first was centred on the transmission of fully-stripped ${ }^{170}$ Dy ions and ran for 13.5 hours of primary beam time [1] with a second setting focused on the transmission of ${ }^{172}$ Dy [10] which ran for 45 hours of primary beam-time. Figure 1 shows the particle identification plot for the transmitted ions centred on the transmission of ${ }^{172} \mathrm{Dy}$ with the ${ }^{166} \mathrm{~Tb}$ species clearly identified. Note that both hydrogen-like $(\mathrm{Z}=\mathrm{Q}-1)$ and fully-stripped $(\mathrm{Z}=\mathrm{Q})$ ions of ${ }^{166} \mathrm{~Tb}$ are transmitted in this particular magnetic rigidity setting.

Figure 2 shows the gamma-ray spectrum gated on ${ }^{166} \mathrm{~Tb}$, with the gamma rays measured between 0.2 and $5.0 \mu \mathrm{s}$ of the implantation of the ions in WAS3ABI. The spectrum shows a discrete transition at energy $119 \mathrm{keV}$

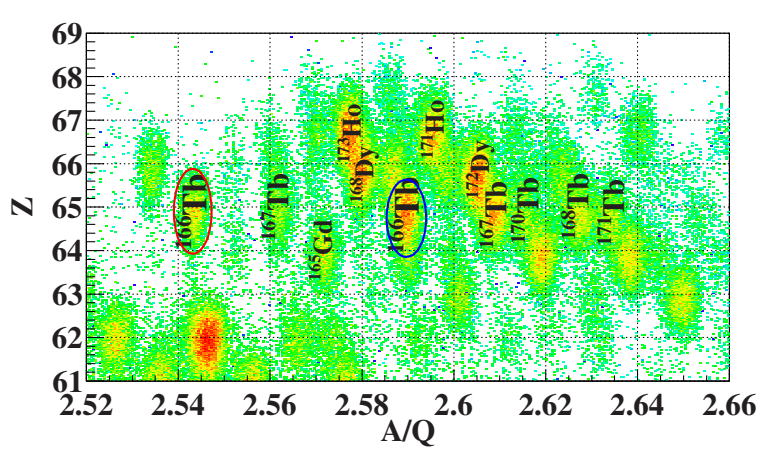

Figure 1. Particle identification plot obtained from the BigRIPS setting centred on ${ }^{172}$ Dy identifying both the hydrogen-like (blue) and fully-stripped (red) ${ }^{166} \mathrm{~Tb}$ ions.

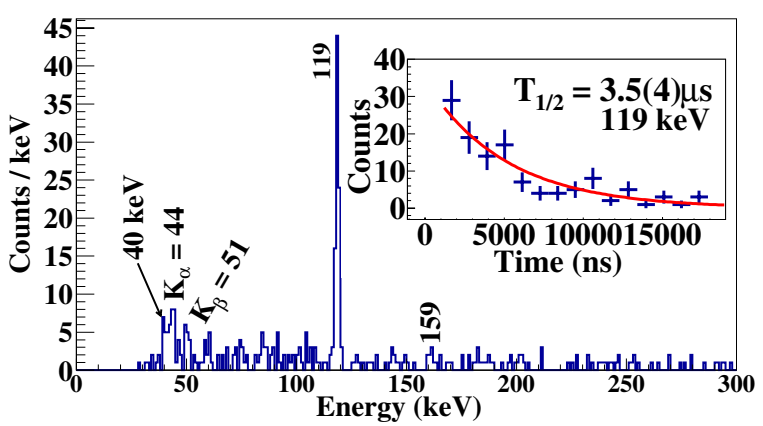

Figure 2. The total projection spectrum of gamma-ray energy from ${ }^{166} \mathrm{~Tb}$ emitted with time condition window from $200 \mathrm{~ns}$ to $5 \mu \mathrm{s}$.

with additional counts associated with the $\mathrm{Tb} \mathrm{K}_{\alpha}$ and $\mathrm{K}_{\beta} \mathrm{X}$ rays. The time distribution of the $119 \mathrm{keV}$ gamma ray relative to the implantation time is shown in the inset of Fig. 2. A single component exponential decay function fit to this decay curve results in a half-life for the isomeric state of 3.5(4) $\mu \mathrm{s}$. We note that the identified gamma-ray transition at $119 \mathrm{keV}$ in the present work was previously noted in the study of the $\beta^{-}$decay of ${ }^{166}$ Dy by Ichikawa et al., [2]. This previous work interpreted the $119 \mathrm{keV}$ transition decaying from an excited state at excitation energy $158 \mathrm{keV}$, with a parallel decay branch with similar intensity associated with a transiton at $158 \mathrm{keV}$. The $119 \mathrm{keV}$ transition was also reported as being in coincidence with a $40 \mathrm{keV}$ gamma ray which fed the proposed ground state of ${ }^{166} \mathrm{~Tb}$. While the $40 \mathrm{keV}$ transition is not clearly separated in the current work, there are counts to the left of the $K_{\alpha}$ X-rays identified in Fig. 2 which are consistent with a transition at that energy. There is also a weakly populated peak in the region $158 \mathrm{keV}$ in Fig. 2, which is consistent with the reported low-lying level scheme for ${ }^{166} \mathrm{~Tb}$ proposed by Ichikawa et al., [2].

Assuming the majority of the branching for the direct decay of the observed isomeric state is via the $119 \mathrm{keV}$ line, its multipolarity can be deduced by comparing the intensity of the terbium $K \mathrm{X}$-rays in the isomer delayed spectrum and the corresponding electromagnetic transition rate for the $119 \mathrm{keV}$ decay. If the $K \mathrm{X}$-rays arise from the competing internal conversion branch of the $119 \mathrm{keV}$ transition from the isomer, this suggests an electric dipole multipolarity for the $119 \mathrm{keV}$ gamma ray, since other likely multipolarities (M1, E2 and M2) all have much higher internal conversion coefficients and would 
Table 1. The calculated internal conversion coefficients, Weisskopf single-particle half-life estimates and transition probabilities assuming a half-life of 3.5(4) $\mu$ s for possible multipolarities of the $119 \mathrm{keV}$ transition in ${ }^{166} \mathrm{~Tb}$. The internal conversion values were estimated using the BRICC code [11].

\begin{tabular}{lllll}
\hline Multipolarity & $\alpha_{\mathrm{K}}(119 \mathrm{keV})$ & $\alpha_{\text {tot }}(119 \mathrm{keV})$ & $\mathrm{T}_{\frac{1}{2}}(1 \mathrm{~W} \cdot \mathrm{u})$ & $B(\sigma \lambda)(\mathrm{Wu})$ \\
\hline E1 & 0.16 & 0.19 & $1.3 \times 10^{-13} \mathrm{~s}$ & $3.8(4) \times 10^{-8} \mathrm{Wu}$ \\
$\mathrm{E} 2$ & 0.72 & 1.39 & $4.3 \times 10^{-7} \mathrm{~s}$ & $1.2(1) \times 10^{-1} \mathrm{Wu}$ \\
M1 & 1.13 & 1.34 & $1.6 \times 10^{-12}$ & $4.5(4) \times 10^{-7} \mathrm{Wu}$ \\
M2 & 8.42 & 11.1 & $4.3 \times 10^{-5}$ & $1.2(1) \times 10^{1} \mathrm{Wu}$ \\
\hline
\end{tabular}

have correspondingly more intense X-ray intensities. The theoretical internal conversion coefficients for the likely multipolarities are given in Table 1, using the BRICC code [11].

The measured half-life of the isomeric state is also most consistent with an E1 decay for the $119 \mathrm{keV}$ transition, with the extracted $\mathrm{B}(\mathrm{E} 1)$ transition probability $3.8(4) \times 10^{-8} \mathrm{Wu}$ consistent with other hindered E1 decays in this deformed region [12].

\section{Possible configurations for the isomeric state in ${ }^{166} \mathrm{~Tb}$}

The low-lying Nilsson configurations in ${ }^{166} \mathrm{~Tb}$ can be investigated by looking at the single quasi-proton and quasi-neutron Nilsson states which have been identified in the neighboring odd-A terbium (for protons) and $\mathrm{N}=$ 101 (for neutrons) nuclei. In general, the lowest-lying Nilsson state, which corresponds to the ground state in the lighter odd- $\mathrm{Z} \mathrm{Tb}$ isotopes is associated with the $\pi \frac{3}{2}^{+}$[411] orbitals. The next lowest-lying excited states associated with intrinsic single-particle states are then linked to the $\pi \frac{7}{2}^{-}$[523], $\pi \frac{5}{2}^{+}$[413] and $\pi \frac{5}{2}^{-}$[532] orbitals respectively [13].

The neutron Nilsson orbitals which are expected to lie closest to the Fermi surface for the prolate deformed, axially symmetric $\mathrm{N}=101$ isotones are the $\frac{1}{2}^{-}[521]$, $\frac{5}{2}^{-}$[512] and $\frac{7}{2}^{+}$[633] states [13], which make up the first three intrinsic states observed in the $\mathrm{N}=101$ isotone ${ }^{169} \operatorname{Er~[14].~The~lowest~lying~expected~two-quasi-particle~}$ states in ${ }^{166} \mathrm{~Tb}$ would then be expected to arise from the coupling of these combinations of proton and neutron Nilsson orbitals, with both maximum and minimum $K$ couplings (i.e., $K=\Omega_{1}+\Omega_{2}$ and $K=\left|\Omega_{1}-\Omega_{2}\right|$ present).

For ${ }^{166} \mathrm{~Tb}$, the lowest-energy configurations, in the absence of residual proton-neutron interactions associated with the Gallagher-Mozkowski coupling rules, would be expected to arise from the $\pi \frac{3}{2}^{+}[411] \otimes \nu \frac{1}{2}^{-}[521]$ configurations, resulting in $K^{\pi}=1^{-}$and $2^{-}$states, one of which is the most likely candidate for the ground state. The $K^{\pi}=1^{-}$is favoured considering the expected residual interaction associated with the anti-aligned intrinsic spins between these two orbitals.

From consideration of the neighboring odd-A isotopes, the next most likely proton-neutron 2 quasi-particle couplings would arise from the $\pi \frac{3}{2}^{+}[411] \otimes v \frac{5}{2}^{-}$[512] configurations which forms $K=1^{-}$and $4^{-}$states, with the maximally aligned $K=4^{-}$favoured by residual interactions; and the $\pi \frac{3}{2}^{+}[411] \otimes v \frac{7}{2}^{+}$[633] which results in $K=2^{+}$and $5^{+}$states, with the $K=5^{+}$favoured. The observed $119 \mathrm{keV}$ E1 isomeric decay could then arise from the $\Delta K=1$ single-particle transition between the $K=5^{+}$
Table 2. The configurations of the low-lying two-quasiparticle states in ${ }^{166} \mathrm{~Tb}$ predicted by the Nilsson blocked BCS calculations, using neutron and proton pairing strengths of $\mathrm{G}_{n}=$ 20.00/A $\cdot \mathrm{MeV}$ and $\mathrm{G}_{p}=21.00 / \mathrm{A} \cdot \mathrm{MeV}$, respectively and quadrupole deformation parameters of $\epsilon_{2}=0.275$ and $\epsilon_{4}=$ 0.027 with axial symmetry $[15,16]$. Note that these calculations do not include any adjustments for residual proton-neutron interactions.

\begin{tabular}{lll}
\hline $\mathrm{K}^{\pi}$ & Nilsson Configuration & Energy, (keV) \\
\hline $2^{-}, 1^{-}$ & $\pi \frac{3}{2}^{+}[411] \otimes v \frac{1}{2}^{-}[521]$ & 0 \\
$3^{-}, 2^{-}$ & $\pi \frac{5}{2}^{+}[413] \otimes \nu \frac{1}{2}^{-}[521]$ & 273 \\
$4^{+}, 3^{+}$ & $\pi \frac{5}{2}^{-}[532] \otimes \nu \frac{1}{2}^{-}[521]$ & 314 \\
$3^{+}, 2^{+}$ & $\pi \frac{5}{2}^{-}[532] \otimes \nu \frac{1}{2}^{-}[521]$ & 355 \\
$5^{+}, 2^{+}$ & $\pi \frac{3}{2}^{+}[411] \otimes v \frac{7}{2}^{+}[633]$ & 62 \\
$6^{+}, 1^{+}$ & $\pi \frac{5}{2}^{+}[413] \otimes v \frac{7}{2}^{+}[633]$ & 335 \\
$7^{-}, 0^{-}$ & $\pi \frac{7}{2}^{-}[523] \otimes v \frac{7}{2}^{+}[633]$ & 376 \\
$6^{-}, 1^{-}$ & $\pi \frac{5}{2}^{-}[532] \otimes v \frac{7}{2}^{+}[633]$ & 417 \\
$4^{-}, 1^{-}$ & $\pi \frac{3}{2}^{+}[411] \otimes v \frac{5}{2}^{-}[512]$ & 145 \\
$5^{-}, 0^{-}$ & $\pi \frac{5}{2}^{+}[413] \otimes v \frac{5}{2}^{-}[512]$ & 418 \\
$6^{+}, 1^{-}$ & $\pi \frac{7}{2}^{-}[523] \otimes v \frac{5}{2}^{-}[512]$ & 459 \\
$5^{+}, 0^{+}$ & $\pi \frac{5}{2}^{-}[523] \otimes \nu \frac{5}{2}^{-}[512]$ & 501 \\
\hline
\end{tabular}

and $K^{\pi}=4^{-}$states from the $\pi \frac{3}{2}^{+}[411] \otimes v \frac{7}{2}^{+}[633]$ to $\pi \frac{3}{2}^{+}[411] \otimes \nu \frac{5}{2}^{-}[512]$ configurations, respectively. The reported energy difference of $159 \mathrm{keV}$ between the $v \frac{5}{2}^{-}$[512] $\left(E_{x}=92 \mathrm{keV}\right)$ and the $v \frac{7}{2}^{+}$[633] $\left(E_{x}=\right.$ $243 \mathrm{keV}$ ) single-particle configurations in the neighboring $\mathrm{N}=101$ isotone ${ }^{169} \mathrm{Er}$ is similar to the observed $119 \mathrm{keV}$ transition in the current work. The current data does not provide any information on the ordering of these possible initial and final states, which could be reversed and would result in the same isomeric decay. In this scenario, the direct decay from either the $K^{\pi}=5^{+}$or $K^{\pi}=4^{-}$ configuration to the proposed negative-parity $K^{\pi}=1^{-}$ ground state via a $40 \mathrm{keV} \mathrm{M} 4$ or M3 decay, which would result in very long-lived metastable state. This is not consistent with the observations in the current work and the previous study of Ichikawa et al., [2] which show the $119 \mathrm{keV}$ and $40 \mathrm{keV}$ transitions in coincidence. A more consistent candidate for the observed E1 decay is between the $K^{\pi}=2^{+}$unfavoured coupling of the $\pi \frac{3}{2}^{+}[411] \otimes$ $v \frac{7}{2}^{+}$[633] configuration and the unfavoured by $K=2^{-}$ coupling of the proposed $\pi \frac{3}{2}^{+}[411] \otimes v \frac{1}{2}^{-}$[521] ground state configuration. This $K=2^{-}$state, could then decay by an unhindered M1 to the favoured $K=1^{-}$coupling of the same configuration, which could correspond to the ground state of ${ }^{166} \mathrm{~Tb}$.

Blocked BCS Nilsson calculations were also performed for ${ }^{166} \mathrm{~Tb}$ using neutron and proton pair- 
ing strengths of $\mathrm{G}_{n}=20.00 / \mathrm{A} \cdot \mathrm{MeV}$ and $\mathrm{G}_{p}=21.00 /$ $\mathrm{A} \cdot \mathrm{MeV}$, respectively [13], together with quadrupole deformation parameters of $\epsilon_{2}=0.275$ and $\epsilon_{4}=0.027$. The results of the calculations are presented in Table 2, with the predicted ground state having the expected $\nu \frac{1}{2}^{-}[521] \otimes \pi \frac{3}{2}^{+}$[411] configuration and the next lowest state being the positive parity $K^{\pi}=5^{+}$and $2^{+}$, $v \frac{7}{2}^{+}[633] \otimes \pi \frac{3}{2}^{+}[411]$ configuration. Note that these calculations do not include any effects associated with residual proton-neutron interactions. The calculations support the possible interpretations described above.

\section{Conclusions}

Isomer-delayed spectroscopy has been performed on the neutron-rich, odd-odd prolate deformed nucleus ${ }^{166} \mathrm{~Tb}$ at the RIBF facility, RIKEN following the production of this isotope via high-energy projectile fission. The data show evidence for the direct decay of an $119 \mathrm{keV}$ electric dipole transition from the isomeric state, which has a measured half-life of 3.5(4) $\mu$ s. Possible Nilsson configurations for the initial and final states which are linked by the $119 \mathrm{keV}$ transition are proposed, based on comparison with neighboring odd-A nuclei and BCSNilsson calculations. The favoured interpretation for the observed, direct $119 \mathrm{keV}$ E1 transition depopulating the isomer is that it arises from the decay between the $K^{\pi}=$ $2^{+}, \pi \frac{3}{2}^{+}[411] \otimes \nu \frac{7}{2}^{+}[633]$ and the $K^{\pi}=2^{-}, \pi \frac{3}{2}^{+}[411] \otimes$ $v \frac{1}{2}^{-}$[521] configurations, the latter of which decays by a $40 \mathrm{keV}$ M1 to the predicted $K^{\pi}=1^{-} \pi \frac{3}{2}^{+}[411] \otimes$ $v \frac{1}{2}^{-}[521]$ ground state configuration.

The authors are indebted to the facility crews who provided the beams at RIBF, operated by RIKEN Nishina Center and CNS, University of Tokyo, the EUROBALL Owners Committee for the loan of germanium detectors, the PreSpec Collaboration for the use of the readout electronics. Part of the WAS3ABi was supported by the Rare Isotope Science Project which is funded by MSIP and NRF of Korea. This work is supported by the UK Science and Technology Facilities Council (STFC); the UK National Measurement Office (PHR); JSPS KAKENHI Grant No. 24740188, 25247045, and 25287065; the U.S.
Department of Energy, Office of Science, Office of Nuclear Physics under Contract No. DE-AC02-06CH11357 (FGK) NRF Korea Grants No.2009-0093817 and 2013R1A1A2063017 (CSL), 2015R1D1A1A01056918, 2016R1A5A1013277 and 2016K1A3A7A09005579 (KYC); the Hungarian Research Fund OTKA contract number K100835 and Science Foundation Ireland under Grant No. 12/IP/1288 (OJR).

\section{References}

[1] P.-A. Söderström et al., Physics Letters B 762, 404 (2016)

[2] S. Ichikawa et al., Physical Review C 71, 067302 (2005)

[3] Y. Yano, Nuclear Instruments and Methods in Physics Research B 261, 1009 (2007)

[4] T. Kubo, Nuclear Instruments and Methods in Physics Research B 204, 97 (2003)

[5] T. Kubo, et al., Progress in Theoretical and Experimental Physics 2012, 03 C003 (2012)

[6] S. Nishimura, Progress in Theoretical and Experimental Physics 2012, 03 C006 (2012)

[7] P.-A. Söderström et al., Nuclear Instruments and Methods in Physics Research B 317, 649 (2013)

[8] Z. Patel et al., RIKEN Accel. Prog. Rep. 47 (2014)

[9] F. Browne et al., Physics Letters B 750, 448 (2015)

[10] H. Watanabe et al., Physics Letters B 760, 641 (2016)

[11] T. Kibédi, T. Burrows, M. Trzhaskovskaya, P. Davidson, and C.W. Nestor. Jr, Nuclear Instruments and Methods in Physics Research A 589, 202 (2008)

[12] Z. Patel et al", Physical Review Letters 113, 262502 (2014)

[13] A.K. Jain, R.K. Sheline, P.C. Sood, and K. Jain, Reviews of Modern Physics 62, 393 (1990)

[14] C.M. Baglin, Nuclear Data Sheets 109, 2033 (2008)

[15] K. Jain, O. Burglin, G. Dracoulis, B. Fabricius, N. Rowley, and P. Walker, Nuclear Physics A 591, 61 (1995)

[16] P. Moller, J. Nix, W. Myers, and W. Swiatecki, Atomic Data and Nuclear Data Tables 59, 185 (1995) 